\title{
Are We Writing a Good Discharge Summary?
}

The discharge summary is a permanent part of a patient's medical record. It is a clinical report prepared by health care providers when a patient is ready for discharge from a hospital or other care facilities. It informs outpatient medical health care providers about services provided by the inpatient care. The discharge summary should include all information pertinent to the ongoing treatment of the patient and the patient's condition. It carries the 'image' of the unit and the hospital. High-quality discharge summaries are essential for promotion of safety of the patient during transitions between care settings, particularly during the initial post-hospital period. ${ }^{1}$

The Joint Commission (TJC), formerly the Joint Commission on Accreditation of Healthcare Organizations (JCAHO) is a United States based non-profit organization. It currently accredits hospitals in Asia, Europe, the Middle East and South America. According to TJC, discharge summaries should include the following elements: the reason for hospitalization, significant findings, procedures performed and care, treatment, and services provided, the patient's condition at discharge and information provided to the patient and family, as appropriate. $^{2}$

In 2012, a study on discharge summary done in a tertiary care hospital in Bangladesh showed that many vital data were missed in the discharge summaries. History of presenting illness was missed in $52 \%$ cases, treatment history in $66 \%$, physical examination findings in $50 \%$, diagnostic test results in $22 \%$, hospital course in $7 \%$, discharge medications in $4 \%$, discharge condition in $48 \%$ and follow-up plans were missed in $44 \%$ cases. $^{3}$ The full name and identity of the person preparing the report was present in $100 \%$ cases. $^{3}$ Omission of information may have a great impact on post-hospital patient care plans, physician practice behavior, post-hospital patient safety and health outcomes. A study conducted in large Midwestern Academic Hospital in USA found that their summaries adhered very well to the components of TJC except the part of 'patients discharge condition'. ${ }^{4}$ Errors related to discontinuity of care occurred in about $50 \%$ of patients and that gaps in communication related to diagnostic evaluations significantly increased the risk of readmissions. ${ }^{5}$

Several studies were performed around the world to evaluate the appropriateness of discharge summary. In our country, most of the hospitals still use handwritten discharge. One important hazard of this type of discharge summary is illegible handwriting. In two separate audits of hospital records, it was found that $20 \%$ of physicians' medication orders ${ }^{6}$ and about $70 \%$ of consultants' operation notes ${ }^{7}$ were illegible. Another study showed that problems still persist for a small but important proportion of records after taking initiatives to improve the legibility of handwriting. ${ }^{8}$ Automated, database-driven reports are the only reasonable alternatives to handwritten summaries. ${ }^{9}$ Time to write discharge note would be less in this way, but it is expensive. A study done in USA revealed that electronic discharge summary significantly improved the timeliness and quality of the summaries. ${ }^{10}$ Another study in Australia agreed with the superiority of electronic discharge over manual discharge as the contents were also written properly. ${ }^{11}$ Electronic summary helps in capturing data process quickly and maintains the integrity of care more efficiently.

It was also noted that physicians do not have any formal training for writing a good summary. Most of the time they learn from their colleagues. There is a preoccupied idea that doctors can write summary without any proper training. Lack of supervision and education leads to poor quality of discharge paper.

In Bangladesh, there is no communication system between primary care physicians and hospitals. Due to the lack of referral system, patients are admitted in hospitals by their own choice and visit local doctors wherever feasible. Thus there is a large gap of communication among the physicians at different levels regarding care of the patients. Often the patients lose their discharge papers and as there is no central database for keeping the documents, all the 
important informations are lost forever. Government and health care sectors should develop a strong network for delivering best health care by improving the infrastructure and forming better communication.

We do not have any national guideline to prepare a good discharge summary. There are also variations of contents of a good summary around the world. A universal guideline should be made for easy, structured and informative discharge summary.

The quality of discharge summary can be improved by health information technology as it would help quick and easy extraction of information regarding diagnoses, medication and investigation results. ${ }^{12}$ Thus a well-structured discharge document can be produced, reviewed and more information regarding specific instructions can be added by physicians.

\section{Rukhsana Parvin}

Associate Professor, Department of Medicine Enam Medical College \& Hospital, Savar, Dhaka

\section{References}

1. Van Walraven C, Seth R, Austin PC, Laupacis A. Effect of discharge summary availability during post-discharge visits on hospital readmission. J Gen Intern Med 2002; 17: 186-192.

2. Standard IM.6.10: Hospital Accreditation Standards. Oakbrook Terrace, III: Joint Commission on Accreditation of Healthcare Organizations; 2006: 338-340.

3. Parvin R, Rafiqueuddin AKM. Are we writing a proper discharge summary? - an observational study in a tertiary care hospital. Paper presented at: The $13^{\text {th }}$ national congress and scientific seminar of Bangladesh Society of Medicine; 2013 March 1-2; Dhaka.

4. Kind AJH, Smith MA. Documentation of mandated discharge summary components in transitions from acute to subacute care. In: Henriksen K, Battles JB, Keyes MA, Grady ML (eds). Advances in patient safety: new directions and alternative approaches (Vol. 2: Culture and redesign). Rockville (MD): Agency for Healthcare Research and Quality (US); 2008. Available at: http://www.ncbi.nlm.nih.gov/books/NBK43715/. Accessed April 2013.

5. Moore C, Wisnivesky J, Williams S, McGinn T. Medical errors related to discontinuity of care from an inpatient to an outpatient setting. J Gen Intern Med 2003; 18: 646-651.

6. Winslow EH, Nestor VA, Davidoff SK, Thompson PG, Borum JC. Legibility and completeness of physicians' handwritten medication orders. Heart Lung 1997; 26: $158-164$.

7. Baigrie RJ, Dowling BL, Birch D, Dehn TC. An audit of the quality of operation notes in two district general hospitals. Are we following Royal College guidelines? Ann R Coll Surg Engi 1994; 76: 8-10.

8. DelMar CB, Lowe JB, Adkins P, Arnold E, Baade P. Improving general practitioner clinical records with a quality assurance minimal intervention. Br J Gen Pract 1998; 48: 1307-1311.

9. Lenhard RE, Buchman JP, Achuff SC, Kahane SN, Macmanus CJ. AUTRES-the Johns Hopkins Hospital automated resume. J Med Syst 1991; 15: 237-247.

10. O'Leary KJ, Liebovitz DM, Feinglass J, Liss DT, Evans $\mathrm{DB}$, Kulkarni $\mathrm{N}$ et al. Creating a better discharge summary: improvement in quality and timeliness using an electronic discharge summary. J Hosp Med 2009; 4(4): 219-225.

11. Alderton M, Callen J. Are general practitioners satisfied with electronic discharge summaries? HIM J 2007; 36(1): 7-12.

12. Branger PJ, van der Wouden JC, Schudel BR, Verboog E, Duisterhout JS, van der Lei J et al. Electronic communication between providers of primary and secondary care. BMJ 1992; 305: 1068-1070. 